\title{
BMJ Open Clinical features, management and outcomes of severe ischaemic stroke in tertiary hospitals in China: protocol for a prospective multicentre registry-based observational study
}

\author{
Simiao Wu, ${ }^{1,2}$ Ruozhen Yuan, ${ }^{1,2}$ Yao Xiong, ${ }^{1,3}$ Shihong Zhang, ${ }^{1,2}$ Bo Wu, ${ }^{1,2}$ \\ Ming Liu ${ }^{1,2}$
}

To cite: Wu S, Yuan R, Xiong Y, et al. Clinical features, management and outcomes of severe ischaemic stroke in tertiary hospitals in China: protocol for a prospective multicentre registry-based observational study. BMJ Open 2018;8:e024900. doi:10.1136/ bmjopen-2018-024900

- Prepublication history and additional material for this paper are available online. To view these files, please visit the journal online (http://dx.doi org/10.1136/bmjopen-2018024900).

Received 21 June 2018 Revised 28 August 2018 Accepted 26 September 2018

Check for updates

(c) Author(s) (or their employer(s)) 2018. Re-use permitted under CC BY-NC. No commercial re-use. See rights and permissions. Published by BMJ.

${ }^{1}$ Department of Neurology, West China Hospital, Sichuan University, Chengdu, China ${ }^{2}$ Centre of Cerebrovascular Diseases, West China Hospital, Sichuan University, Chengdu, China

${ }^{3}$ Department of Neurology, The Third People's Hospital of Chengdu, Chengdu, China

Correspondence to

Professor Ming Liu;

wyplmh@hotmail.com

\section{ABSTRACT}

Introduction Severe ischaemic stroke is a devastating condition with high mortality and morbidity; however, there is insufficient evidence on its management. The aim of this study is to investigate causes, risk factors, clinical course, management and outcomes of severe ischaemic stroke in a real-world setting in tertiary hospitals in China.

Methods and analysis This is a prospective, multicentre, registry-based observational study. We will recruit 2500 patients with acute ischaemic stroke from nine tertiary hospitals in Western China. Patients with acute ischaemic stroke admitted to the Department of Neurology within 30 days of stroke onset will be included. Patients will be visited within 24 hours after admission, on day 3 , day 7 and at discharge, to collect data on their clinical state, blood biomarkers and brain imaging. Severe stroke is defined as severe neurological deficits (National Institute of Health Stroke Scale (NIHSS) $\geq 15$ or in coma) on admission or clinical worsening (NIHSS increased by $\geq 4$ scores) during hospitalisation. Patients will be followed up by structured telephone interviews at 3 months and 1 year after stroke onset. In-hospital outcomes include symptomatic haemorrhagic transformation and brain oedema by day 7 of admission, and survival status (death or survival) by discharge; follow-up outcomes will include survival status and functional outcome (assessed by modified Rankin Scale) at 3 months and 1 year. The current study will improve our knowledge about the development of severe ischaemic stroke at acute phase and factors influencing its outcomes, which will eventually facilitate optimisation of individualised interventions for its prevention and treatment. Ethics and dissemination Ethics approval is obtained from The Biomedical Research Ethics Committee of West China Hospital, Sichuan University (Reference No. 2017(130)). We will present our findings at the national and international conferences and peer-reviewed journals in stroke and neurology.

Trial registration number NCT03222024; Pre-results.
Strengths and limitations of this study

- This is a large multicentre registry-based prospective cohort study to systematically investigate clinical features, management and outcomes of patients with severe ischaemic stroke in tertiary hospitals in China.

- We chose tertiary hospitals because most patients with severe ischaemic stroke in China are cared in these hospitals, which would reflect a real-world practice pattern.

- Another strength is that we used a comprehensive definition of severe stroke, incorporating initial neurological function and clinical worsening during hospitalisation, to better cover patients requiring intensive management in acute phase.

- We provided intensive in-hospital assessments and long-term follow-up to understand natural history of severe ischaemic stroke.

- As of common limitations to observational studies, our study may subject to selection bias and inaccurate data recording. To overcome such limitations, we provided regular training and monitoring to site researchers to promote adherence to protocol and ensure Good Clinical Practice.

\section{INTRODUCTION}

Acute ischaemic stroke is a leading cause of mortality and morbidity in the world. About one-third of patients recruited in trials of intravenous thrombolysis for acute ischaemic stroke presented with severe neurological deficits, known as severe ischaemic stroke. ${ }^{12}$ Severe stroke is usually related to large infarction in middle cerebral artery (MCA) territories, termed as large hemispheric infarction $(\mathrm{LHI})^{3}$ or massive MCA infarction, ${ }^{4}$ and is associated with poor outcomes at short term ${ }^{5}$ and long term. ${ }^{6}$ In addition, brainstem infarction can be fatal even the lesion could be relatively small. Apart from initially severe stroke, 
malignant MCA infarction is another type of severe stroke associated with a high risk of death. It is a clinical syndrome characterised by a rapid increase in the severity of neurological deficits associated with cerebral oedema, which results in massive hemispheric brain swelling, with or without haemorrhagic transformation, leading to transtentorial herniation, death or very poor functional outcomes. $^{7}$

Despite its devastating consequences, little evidence is available to inform the prevention and management of patients with either initially severe or malignant-course strokes. Although a number of interventions are recommended for general management of acute ischaemic stroke, we do not know which patients with very severe stroke benefit most from conventional stroke-specific therapies such as thrombolysis, ${ }^{8}$ antiplatelet agents ${ }^{9}$ and anticoagulants. ${ }^{10}$ Due to this gap of knowledge, international guidelines have been published for management of $\mathrm{LHI}^{3}$ and malignant stroke with oedema ${ }^{11}$; however, most of the clinical recommendations are derived from general stroke guidelines as patients with severe stroke are often excluded from clinical trials investigating interventions for stroke. ${ }^{12}$ In addition, there are insufficient data on the effect of hypothermia, ${ }^{13}$ corticosteroids ${ }^{14}$ and osmotic therapy ${ }^{15}$ for the treatment of stroke and certainly insufficient in severe stroke, despite their frequent use in practice. ${ }^{11}$ Three randomised controlled trials, Hemicraniectomy After Middle Cerebral Artery infarction with Life-threatening Edema Trial (HAMLET) ${ }^{16}$ Decompressive Surgery for the Treatment of Malignant Infarction of the Middle Cerebral Artery Trial (DESTINY) ${ }^{17}$ and Early Decompressive Craniectomy in Malignant Middle Cerebral Artery Infarction Trial (DECIMAL), ${ }^{18}$ investigated decompressive hemicraniectomy (DHC) for patients with supratentorial infarction and all reported that DHC reduced mortality as compared with medical management. However, as the benefit of DHC is evident only in highly selected patients and the improved survival is gained at the cost of persistent morbidity in many survivors, DHC is underused in practice despite the recommendation in clinical guidelines. ${ }^{311}$

Lack of effective interventions is partly due to our limited knowledge on mechanisms that underpin the malignant evolution of stroke in some patients. Few studies have systematically investigated either modifiable risk factors for the development of stroke worsening or those affecting the prognosis of severe stroke. A systematic review of predictors of life-threatening brain oedema in MCA infarction was published in 2008, which included 23 observational studies with 1185 patients and identified 27 factors associated with brain oedema; however, even the best predictor reported in this review has only moderate predictive value. ${ }^{19}$ It is noticeable that all included studies are small and may lack of power to detect predictive value. So far the largest observational study on severe stroke, derived from the Acute Stroke Registry and Analysis of Lausanne, was published in 2012. ${ }^{5}$ By comparing 243 patients with stroke with an initial National Institute of
Health Stroke Scale (NIHSS) score $\geq 20$ with 1672 patients with NIHSS $<20$, the study found that the presence of severe stroke on admission was associated with multiple clinical factors, brain imaging and blood biomarkers; in addition, favourable outcome at 3 months of patients with initially severe stroke was predicted by several modifiable factors. ${ }^{5}$ Early identification of these risk factors and interventions for modifiable factors would be useful for the prediction and management of severe stroke. However, this study used an 'arbitrary' cut-off score of NIHSS $\geq 20$ on admission to define 'severe stroke', which may not fully reflect clinical features of this complex condition. In addition, the study did not investigate factors associated with the evolution from mild stroke to severe stroke in hospital, which is a very important issue for acute stroke management.

In summary, there are uncertainties in the causes, risk factors, clinical course, management and outcomes of severe ischaemic stroke, thus, this multicentre prospective cohort study is designed to investigate into these areas in a real-world setting in tertiary hospitals in China. Particularly, as malignant brain oedema is based on our clinical observation, possibly the most common cause of neurological worsening following acute ischaemic stroke, we will explore predictors for its evolution and strategies specifically targeting its prevention and treatment.

\section{METHODS AND ANALYSIS}

This is a multicentre, registry-based, prospective cohort study, which will start in March 2017 and end in February 2020. We will recruit patients with acute ischaemic stroke admitted to the Department of Neurology of nine participating hospitals (details in the Acknowledgements section). Appropriate local ethics committee approvals are sought for each participating hospital and proof of local approval must be sent to the leading centre before recruitment can be started in each centre. The study must be run according to local law and regulation. Figure 1 is a summary of study processes. The reporting of this protocol complies with the Standard Protocol Items: Recommendations for Interventional Trials recommendation (online supplementary file 1).

\section{Patient and public involvement}

Patients and public were not involved in the design of the study. Participation of patients with ischaemic stroke will provide data to answer the research questions of current study. We will disseminate the study findings to patients with stroke in our routine practice in neurology wards and clinics, and through public education activities and stroke academic conferences.

\section{Participants}

Inclusion and exclusion criteria

In each participating hospital, patients with stroke admitted to the Department of Neurology will be screened for eligibility by their responsible doctors. Inclusion 


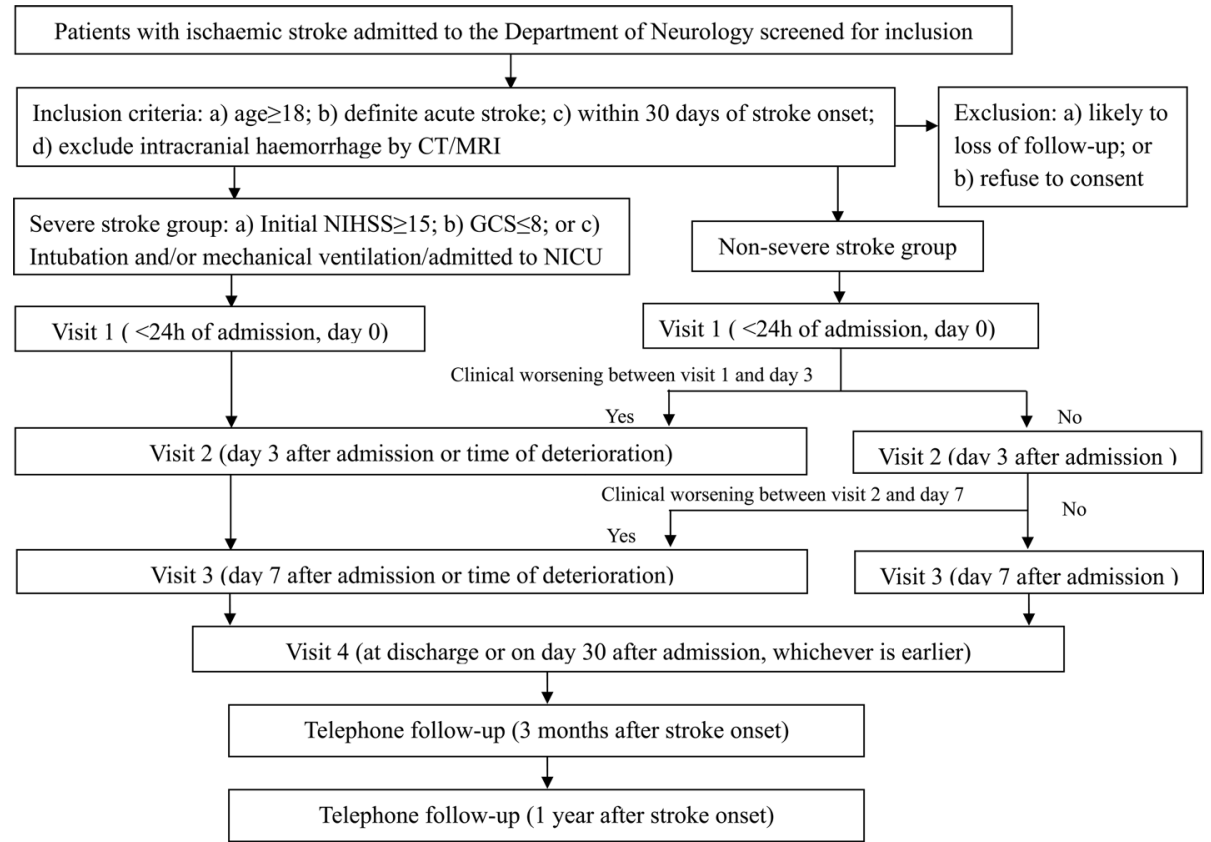

Figure 1 Flow chart of study processes for clinical features, management and outcomes of severe ischaemic stroke. GCS, Glasgow Coma Scale; NIHSS, National Institute of Health Stroke Scale; NICU, neurological intensive care unit.

criteria: (1) Aged 18 years or over; (2) Symptoms and signs of clinically definite acute stroke; (3) Time of stroke onset is known and within 30 days of admission and (4) CT or MRI brain scanning has reliably excluded both intracranial haemorrhage and structural brain lesions which can mimic stroke (eg, brain tumour). Exclusion criteria: (1) Likely to be unavailable for follow-up, for example, no fixed home address or (2) Refuse to give consent to participate. All patients (or their legal proxies) will be provided with a written patient information sheet and be fully informed the aim and content of this study. Each patient (or their proxies if the patient is incapable in decision making) will be asked to sign an informed consent form before they are recruited.

\section{Definition of severe ischaemic stroke}

We searched PubMed with search terms (stroke OR ischemi* OR ischaemi* OR infarct OR infarction) AND (severe OR malignant OR massive OR large OR hemispheric OR edema OR oedema OR swelling OR herniation OR space-occupying) for clinical trials, observational studies and reviews. We summarised commonly reported definitions of 'severe stroke' and had a panel discussion with doctors and nurses working in stroke wards and neurological intensive care unit (NICU) of participating hospitals, and reached consensus on the following operational definition of 'severe stroke' to be used in the current study: (1) severe neurological deficits, assessed by the NIHSS scored 15 or over, (2) loss of consciousness, assessed by the Glasgow Coma Scale (GCS) scored 8 or less, or item 1a of NIHSS scored 1 or over, or (3) intubation, mechanical ventilation or admitted to NICU for any reason on admission or during current hospitalisation for ischaemic stroke.
We define our patients as three groups: (1) initially severe stroke: patients who fulfil the above prespecified operational definition of severe stroke on admission; (2) late-developed severe stroke (ie, malignant-course stroke): patients who do not initially fulfil the definition of severe stroke on admission, but experience clinical worsening and develop severe stroke within 7 days after stroke onset and (3) non-severe stroke: patients do not have either initially severe stroke or late-developed severe stroke. We define clinical worsening as (1) neurological deterioration with an increase of NIHSS score of 4 or more as compared with baseline NIHSS, (2) a decline of consciousness, which leads to a GCS score of 8 or less, or item 1a of NIHSS scored 1 or over, (3) need for invasive interventions such as DHC or (4) death in hospital.

\section{In-hospital visits and data collection}

In-hospital visits will be performed by on-site neurologists or trained postgraduate students in the Department of Neurology in each participating hospital. After patients (or their proxies) sign the consent form, we will collect patients' baseline data within 24 hours after admission (visit 1, day 0). Subsequent visits will be conducted on day 3 after admission or earlier if the patient experiences clinical worsening (as defined above) at any time between day 0 and day 3 (visit 2), on day 7 after admission or earlier if clinical worsening occurs at any time between visit 2 and day 7 (visit 3), and on the day before discharge or on day 30 after admission, whichever is earlier (visit 4 , the average hospital stay for patients with ischaemic stroke is about 2 weeks, and can be longer for some patients with severe stroke). If the patient dies or is discharged before a planned visit, visit 4 will be conducted accordingly with the planned visit omitted. 
Table 1 Data collection during hospitalisation and up to 1 year after stroke onset

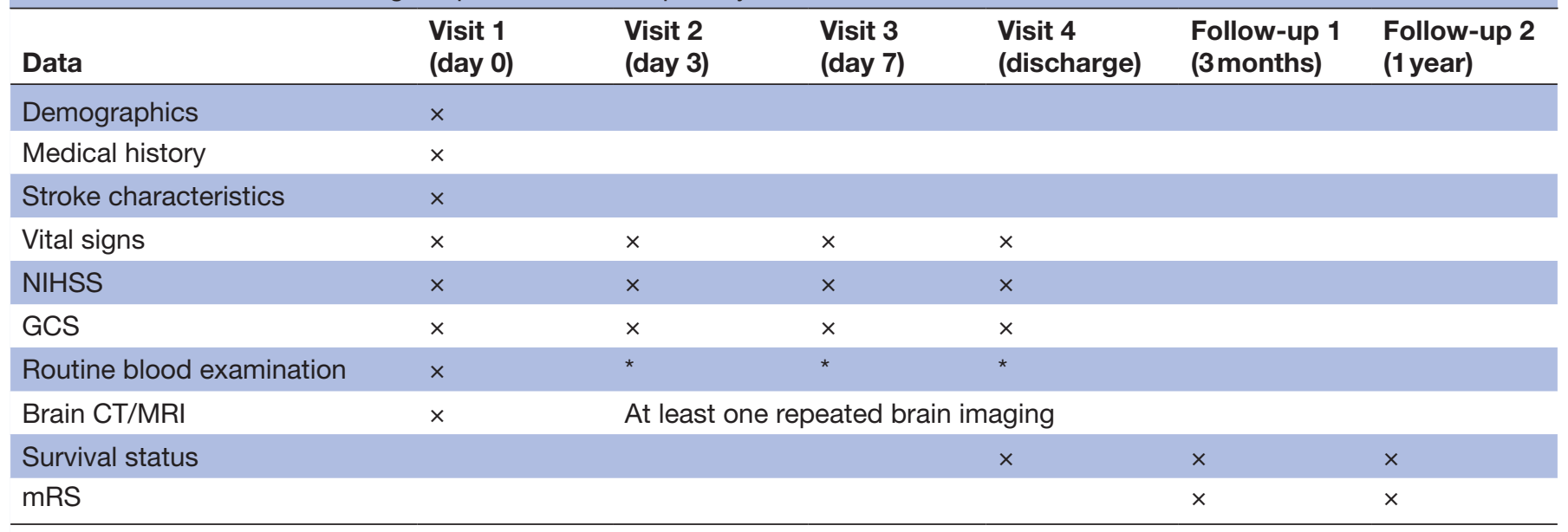

$\times$ Required; *if applicable.

NIHSS, National Institute of Health Stroke Scale; GCS, Glasgow Coma Scale; mRS: modified Rankin Scale.

A paper-based structured case report form will be used to record information for each patient. Data collected at each visit are summarised in table 1 . Particularly, at visit 1, we will record demographics (age and sex), medical history (eg, prestroke disability (assessed by modified Rankin Scale, mRS), hypertension (blood pressure consistently above $140 / 90 \mathrm{~mm} \mathrm{Hg}$ or treated hypertension on medication), diabetes mellitus, atrial fibrillation, history of stroke), clinical characteristics of current stroke (eg, initial stroke severity by NIHSS, consciousness level by GCS), results of routine blood tests on admission (eg, white cell count, renal function indices including serum creatinine concentration), and stroke features on initial brain imaging (site and size of cerebral infarction, extent of cerebral swelling and presence of haemorrhagic transformation according to criteria by Wardlaw and Sellar).$^{20}$ For subsequent visits, we will assess stroke characteristics (eg, change in stroke severity by NIHSS, change of consciousness level by GCS) and brain imaging features (eg, brain swelling, haemorrhagic transformation) and record current medical treatment for each patient (eg, thrombolysis, endovascular interventions, antiplatelet agents, anticoagulation, statins, anticerebral oedema therapies, DHC). If the patient has an examination (eg, brain CT) more than once between two adjacent visits, results of the most recent examination to the latter visit will be recorded. At visit 4, we will record treatment interventions used during hospitalisation. We will also record if the patient is referred to neurosurgery department for DHC or to rehabilitation department for further rehabilitation.

\section{Follow-up assessments}

At 3 months and 1 year after stroke onset, a trained researcher (a neurologist or postgraduate in neurology) blind to all relevant medical information will contact the patient to deliver a structured telephone interview and collect the data on survival status (survival or death and cause for death) and functional outcome (assessed by scores on mRS) (table 1).

\section{Clinical outcomes}

In-hospital outcomes include symptomatic haemorrhagic transformation (defined as significant clinical worsening accompanied by clear evidence of significant intracranial haemorrhage on the brain scan) and symptomatic brain oedema (defined as significant clinical worsening accompanied by evidence of significant brain swelling including shift of the midline away from the side of the ventricle or effacement of the basal cisterns or uncal herniation on the brain scan) by day 7 of admission, and survival status (death or survival) by discharge; follow-up outcomes include survival status and functional outcome (assessed by $\mathrm{mRS}$ ) at 3 months and 1 year.

\section{Provisional analysis plan}

We have planned the following analyses:

A. Clinical features, management and outcomes of patients with severe ischaemic stroke.

All patients will be included in this analysis. Comparisons will be performed between patients with initially severe stroke, patients with late-developed severe stroke and those without severe stroke, for their demographics, medical history, stroke characteristics, in-hospital interventions, and survival and functional outcomes at discharge, 3 months and 1 year. Particularly, clinical course of neurological deficits will be described for patients who develop severe stroke, for subgroups of patients admitted within 24 hours, 2-7 days and after 7 days of stroke onset.

B. Prognostic factors for patients with severe ischaemic stroke.

Patients with initially severe stroke and those with late-developed severe stroke will be included in this analysis. Comparisons will be performed between patients with good functional outcomes $(\mathrm{mRS} \leq 2)$ with 
those with poor functional outcomes $(\mathrm{mRS} \geq 3)$ at 3 months, for their demographics, medical history, stroke characteristics and in-hospital interventions. Subgroup analyses will be performed for the time of admission, comparing patients admitted within 24 hours, $2-7$ days and beyond 7 days.

C. Causes for clinical worsening within 7 days after stroke onset, and risk factors and outcomes of patients with each cause.

Patients admitted within 24 hours after stroke onset will be included for this analysis. Comparisons will be performed between patients developing clinical worsening within 7 days after onset and those not developing clinical worsening, and further between patients with each cause of clinical worsening. Possible causes include but are not limited to cerebral events such as brain swelling, intracranial haemorrhage and recurrent stroke, and non-cerebral events such as pulmonary infection, myocardial infarction and extracranial bleeding. Patients with each cause of clinical worsening and those without clinical worsening will be compared for demographics, medical history, clinical and radiological characteristics of current stroke, interventions and outcomes at discharge, 3 months and 1 year. For the subgroup of patients who develop malignant brain oedema (ie, initially severe stroke or late-developed severe stroke, with imaging evidence of brain swelling) compared with patients without malignant brain oedema, we will perform multivariate analysis to explore clinical and radiological factors that independently predict the development of malignant brain oedema.

\section{Statistical analyses}

Data will be described in mean (SD) or median (range) for continuous variables and counts (percentages) for categorical variables. Two group comparisons will be conducted by independent Student's t-test or MannWhitney $\mathrm{U}$ test for continuous variables and $\chi^{2}$ test or Fisher's exact test for dichotomous variables. Associations between potential risk factors and outcomes will be assessed by ORs and relevant 95\% CIs. Multivariate logistic regression will be used to investigate the effects of potential risk factors on the development malignant brain oedema. Cox proportional hazard model will be used to explore factors affecting prognosis at 3 months and 1 year. We will perform multiple imputation to account for missing data and also perform sensitivity analysis by excluding patients with missing data.

\section{Sample size estimation}

This is an observational study with a primary aim to describe the clinical features and outcomes of patients with severe ischaemic stroke, which would often not require sample size estimation. Therefore, we calculate the sample size for the logistic analysis for predicting malignant brain oedema, for which we anticipate to test $5-10$ variables as potential predictors. Based on the criteria of at least 10 events per variable, ${ }^{21}{ }^{22}$ we will need $50-100$ patients with malignant brain oedema. Assuming a proportion of $4 \%$ of general patients with ischaemic stroke would develop malignant brain oedema, ${ }^{1}$ a sample of $1250-2500$ patients is required. In this observational study, we aim to recruit 2500 patients in order to allow a possible withdrawal rate of $10 \%$ and provide sufficient information on different subgroups of patients for further analyses.

\section{Data management}

Site data will be collected by responsible doctors at each participating hospital and sent securely to the central office in West China Hospital, Sichuan University. Researchers of the central management team and relevant regulatory authorities will have access to the information, for which the consent will be sought from each patient at the very beginning of the study. Paperwork with personal information will be stored in a locked filing cabinet in the central office. Clinical data recorded on case report forms will be entered to an electronic database (IBM SPSS, V.21.0, IBM). Study data will be analysed and published in the anonymised form.

\section{Data and safety monitoring}

The steering committee consists of the principal investigator (ML, committee chair) based on West China Hospital, Sichuan University (the leading research centre) and chief investigators of eight participating hospitals (FG, DY, XL, BW, CW, JD, HZ, TL; details in the Acknowledgements section). The committee will be responsible for overseeing the conduct of study to ensure compliance with Good Clinical Practice guidelines and the study protocol. Interim analyses will be performed annually to assess the adherence of study administration to protocol and the progress of study conduction. The committee will advise the central management team on necessary modifications or discontinuation of the study.

The central management team (ML, SW, RY, SZ, BW) based on the leading research centre is responsible for all aspects of day to day management of the study, including coordinating the recruitment of participating hospitals, providing training and study materials, organising training and study meetings, conducting central data collection and checking, performing data analysis and coordinating the production of study reports and publications. With advice on study design modification from the steering committee, the central management team will report to the ethics committee and obtain the approval before implementing the changes. Ethics committee, which is independent from investigators and the sponsor of this study, will perform annual audits for the study conduct.

\section{DISCUSSION}

This paper describes the design of a multicentre, registry-based, prospective observational study, which provides a real-world setting to explore causes, risk factors, clinical course, management and outcomes of severe ischaemic 
stroke. To our knowledge, this will be the largest observational study that systematically investigate severe ischaemic stroke. By exploring causes and clinical course of severe stroke including the development of late-developed severe stroke, this study will inform when severe stroke is likely to occur and why. These findings will suggest the timing for intensive monitoring for patients at risk and optimal strategies for treatment. This study will also provide information on which patients with severe ischaemic stroke could benefit from conventional therapies of stroke management, thus optimise individualised treatment for patients with stroke with severe neurological deficits. Furthermore, by investigating factors existing prior to stroke and in acute phase (including in-hospital interventions) that are associated with the subsequent malignant brain oedema, we will be able to focus on modifiable factors as they are potential targets for preventive interventions. In addition, we will analyse factors that influence the effects of available treatment strategies for malignant brain oedema, with an aim to find out individuals who may benefit from certain intervention. In summary, the current study will improve our knowledge of the development of severe ischaemic stroke at acute phase and factors influencing its outcomes, which will eventually facilitate optimisation of individualised interventions for its prevention and treatment.

\section{Limitations}

As a prospective cohort study, current study cannot avoid common limitations of observational studies such as selection bias and inadequacy of data recording. To overcome these possible limitations, we select tertiary hospitals and require researchers to have clinical and research experience in stroke, in order to ensure a similar high quality of medical services and Good Clinical Practice. We also conduct regular site monitoring and researcher training meetings twice per year, to promote adherence to protocol. We only include tertiary hospitals, because such hospitals are where most patients with stroke will be admitted, thus could reflect a real-world setting of stroke care in China.

\section{ETHICS AND DISSEMINATION}

Appropriate local ethics committee approvals are sought for each participating hospital and proof of local approval must be sent to the leading centre before recruitment can be started in each centre. All patients will be fully informed of the study with written information and will be asked to sign the consent form before they are recruited. We will present our findings at the national and international conferences and peer-reviewed journals in stroke and neurology.

Acknowledgements Nine tertiary hospitals in Western China, including five teaching hospitals, are participating in the study: West China Hospital, Sichuan University; Sichuan Academy of Medical Sciences \& Sichuan Provincial People's Hospital; Affiliated Hospital Of Southwest Medical University; Affiliated Hospital of North Sichuan Medical College; Chengdu University of Traditional Chinese Medicine;
Mianyang Central Hospital; People's Hospital of Deyang City; The First People's Hospital of Ziyang and Jiangyou People's Hospital. We would like to thank Peter Sandercock, University of Edinburgh, who provides advice on study design of this study and comments on the manuscript of this protocol. A number of people have already contributed to the development of the study, particularly clinical and research staff in participating hospitals who support the study, without whom the study would not be possible. We would like to thank chief investigators of eight participating hospitals: Fuqiang Guo (Sichuan Academy of Medical Sciences \& Sichuan Provincial People's Hospital), Xiaogang Li (Affiliated Hospital of Southwest Medical University), Bihua Wu (Affiliated Hospital of North Sichuan Medical College), Dongdong Yang (Affiliated Hospital of Chengdu University of Traditional Chinese Medicine), Jingfeng Duan (Mianyang Central Hospital), Chun Wang (People's Hospital of Deyang City), Tianjin Ling (The First People's Hospital of Ziyang) and Hao Zhang (Jiangyou People's Hospital).

Contributors $M L$ and SW conceived and designed the study. ML is the grant holder. SW, RY and YX conducted the systematic review of literature on severe stroke that underpinned the rationale of the current study. SZ and BW provided advice on clinical and imaging issues. SW wrote the first draft and all authors critically appraised the protocol. All authors have read and approved the final manuscript.

Funding This work was supported by Major International (Regional) Joint Research Project, National Natural Science Foundation of China (Grant No. 81620108009) and Key Research and Development Programme, Science \& Technology Department of Sichuan Province (Grant No. 2017SZ0007).

Disclaimer Study sponsor and funders play no role in design and management of the study, collection, analysis and interpretation of data, writing of the report or the decision on submitting the report for publication.

Competing interests None declared.

Patient consent Obtained.

Ethics approval Central ethics approval is obtained from The Biomedical Research Ethics Committee of West China Hospital, Sichuan University (Reference No. 2017(130)).

Provenance and peer review Not commissioned; externally peer reviewed.

Open access This is an open access article distributed in accordance with the Creative Commons Attribution Non Commercial (CC BY-NC 4.0) license, which permits others to distribute, remix, adapt, build upon this work non-commercially, and license their derivative works on different terms, provided the original work is properly cited, appropriate credit is given, any changes made indicated, and the use is non-commercial. See: http://creativecommons.org/licenses/by-nc/4.0/.

\section{REFERENCES}

1. Sandercock P, Wardlaw JM, Lindley RI, et al. The benefits and harms of intravenous thrombolysis with recombinant tissue plasminogen activator within $6 \mathrm{~h}$ of acute ischaemic stroke (the third international stroke trial [IST-3]): a randomised controlled trial. Lancet 2012;379:2352-63.

2. Wahlgren N, Ahmed N, Dávalos A, et al. Thrombolysis with alteplase for acute ischaemic stroke in the Safe Implementation of Thrombolysis in Stroke-Monitoring Study (SITS-MOST): an observational study. Lancet 2007;369:275-82.

3. Torbey MT, Bösel J, Rhoney DH, et al. Evidence-based guidelines for the management of large hemispheric infarction : a statement for health care professionals from the Neurocritical Care Society and the German Society for Neuro-intensive Care and Emergency Medicine. Neurocrit Care 2015;22:146-64.

4. Kasner SE, Demchuk AM, Berrouschot J, et al. Predictors of fatal brain edema in massive hemispheric ischemic stroke. Stroke 2001;32:2117-23.

5. Bill O, Zufferey P, Faouzi M, et al. Severe stroke: patient profile and predictors of favorable outcome. J Thromb Haemost 2013;11:92-9.

6. Hankey GJ. Long-term outcome after ischaemic stroke/transient ischaemic attack. Cerebrovasc Dis 2003;16:14-19.

7. Huttner HB, Schwab S. Malignant middle cerebral artery infarction: clinical characteristics, treatment strategies, and future perspectives. Lancet Neurol 2009;8:949-58.

8. Whiteley WN, Thompson D, Murray G, et al. Targeting recombinant tissue-type plasminogen activator in acute ischemic stroke based on risk of intracranial hemorrhage or poor functional outcome: an analysis of the third international stroke trial. Stroke 2014;45:1000-6. 
9. Thompson DD, Murray GD, Candelise L, et al. Targeting aspirin in acute disabling ischemic stroke: an individual patient data meta-analysis of three large randomized trials. Int $J$ Stroke 2015;10:1024-30.

10. Whiteley WN, Adams HP, Bath PM, et al. Targeted use of heparin, heparinoids, or low-molecular-weight heparin to improve outcome after acute ischaemic stroke: an individual patient data meta-analysis of randomised controlled trials. Lancet Neurol 2013;12:539-45.

11. Wijdicks EF, Sheth KN, Carter BS, et al. Recommendations for the management of cerebral and cerebellar infarction with swelling: a statement for healthcare professionals from the American Heart Association/American Stroke Association. Stroke 2014;45:1222-38.

12. Lorenzano S, Toni D. TESPI trial Investigators. TESPI (Thrombolysis in Elderly Stroke Patients in Italy): a randomized controlled trial of alteplase (rt-PA) versus standard treatment in acute ischaemic stroke in patients aged more than 80 years where thrombolysis is initiated within three hours after stroke onset. Int J Stroke 2012;7:250-7.

13. Den Hertog HM, van der Worp HB, Tseng MC, et al. Cooling therapy for acute stroke. Cochrane Database Syst Rev 2009:Cd001247.

14. Sandercock PA, Soane T. Corticosteroids for acute ischaemic stroke. Cochrane Database Syst Rev 2011:Cd000064.

15. Bereczki D, Fekete I, Prado GF, et al. Mannitol for acute stroke. Cochrane Database Syst Rev 2007:Cd001153.
16. Hofmeijer J, Kappelle LJ, Algra A, et al. Surgical decompression for space-occupying cerebral infarction (the Hemicraniectomy After Middle Cerebral Artery infarction with Life-threatening Edema Trial [HAMLET]): a multicentre, open, randomised trial. Lancet Neurol 2009;8:326-33.

17. Jüttler $E, S c h w a b S$, Schmiedek $P$, et al. Decompressive Surgery for the Treatment of Malignant Infarction of the Middle Cerebral Artery (DESTINY): a randomized, controlled trial. Stroke 2007;38:2518-25.

18. Vahedi K, Vicaut E, Mateo J, et al. Sequential-design, multicenter, randomized, controlled trial of early decompressive craniectomy in malignant middle cerebral artery infarction (DECIMAL Trial). Stroke 2007;38:2506-17.

19. Hofmeijer J, Algra A, Kappelle LJ, et al. Predictors of life-threatening brain edema in middle cerebral artery infarction. Cerebrovasc Dis 2008;25:176-84.

20. Wardlaw JM, Sellar R. A simple practical classification of cerebral infarcts on CT and its interobserver reliability. AJNR Am J Neuroradiol 1994;15:1933-9.

21. Harrell FE, Lee KL, Mark DB. Multivariable prognostic models: issues in developing models, evaluating assumptions and adequacy, and measuring and reducing errors. Stat Med 1996:15:361-87.

22. Peduzzi P, Concato J, Kemper E, et al. A simulation study of the number of events per variable in logistic regression analysis. $J$ Clin Epidemiol 1996;49:1373-9. 